\title{
Stability limits in double stars
}

\section{A study of inclined planetary orbits}

\author{
E. Pilat-Lohinger, B. Funk, and R. Dvorak
}

Institute for Astronomy, University of Vienna, Türkenschanzstrasse 17, 1180 Vienna, Austria

Received 25 July 2002 / Accepted 12 November 2002

\begin{abstract}
We treat the problem of the stability of planetary orbits in double stars with the aid of numerical studies in the model of the elliptic restricted three-body problem. Many investigations exist for the limits of the stability of the S-type planetary motion (i.e. the motion around one component of the binary). In this paper we present a numerical investigation of the so-called P-type orbits which are defined as orbits of the planet around both primary masses. The growing interest of such stability studies is due to the discovery of the numerous extra-solar planets, where five of them move in double star systems. Two different methods are used; in a first study the stability was investigated systematically for different initial conditions of the planet, which is regarded to be massless. We vary the initial starting distance from the barycenter (always with velocities corresponding to circular orbits at the beginning), the angle to the connecting line of the binary and the starting position of the primaries (peri-astron and apo-astron); the primaries' masses are considered to be equal. The numerical stability criterion is set to the condition that during the whole integration time (50000 periods of the primaries) the planet should not suffer from a close encounter to one of the primaries. The binary's eccentricity $(e)$ is increased form 0 to 0.5 with $\Delta e=0.05$, and the initial distance (A) to the barycenter is taken of the range from 1.8 to $3.5 \mathrm{AU}$ (for $e \leq 0.15$ ) or to $4.5 \mathrm{AU}$ (for $e \geq 0.2$ ) with $\Delta \mathbf{A}=0.05 \mathrm{AU}$. For the first time, we study inclined P-type orbits $\left(0^{\circ} \leq i \leq 50^{\circ}\right.$ with a step of $\left.\Delta i=2.5^{\circ}\right)$ for which we analyse the border line of the stable zone. Additionally we also record the escape times for all orbits. In the second part of our study we apply the Fast Lyapunov Indicator $(F L I)$ to distinguish between the differnt types of motion, taking the same initial conditions, only the grid for the initial distance to the barycenter was finer $(\triangle \mathbf{A}=0.01 \mathrm{AU})$. Since the $F L I$ is known to be a fast chaos indicator we reduced the integration time to $10^{4}$ periods of the primaries. A comparison of the results of the two studies show that they are in good agreement. It turned out that the inclination does not influence the stability significantly. The stability limit itself varies between $\mathbf{A}=2.1 \mathrm{AU}$ (for $e=0$ and $i=42,5^{\circ}$ ) and $\mathbf{A}=3.85 \mathrm{AU}$ (for $e=0.5$ and $i=50^{\circ}$ ).
\end{abstract}

Key words. stars: binaries: general - stars: planetary systems

\section{Introduction}

Until now more than 100 extra-solar planets ${ }^{1}$ have been discovered; most of them are single-planetary systems. We find (a) very massive planets (from 0.12 to 11 Jupiter-masses; note that only $1 / 3$ of the planets have masses $\leq m_{\text {Jupiter }}$ ), (b) planets which are very close to their host-stars (more than $1 / 3$ move in orbits closer than Mercury around the Sun) and (c) high eccentric orbits (about half of the planets move in orbits with eccentricities $\geq 0.3$ ). We did not yet discover an extra-solar planetary system similar to our solar system, even if some planets have been found in the so-called habitable zone. All of them are giant planets moving in high eccentric orbits, except HD 28185 which has an eccentricity of only 0.06 but more than 5 Jupitermasses. However, recently Marcy et al. (2002) discovered the first giant planet moving at about 5.5 AU (which can be

Send offprint requests to: E. Pilat-Lohinger, e-mail: lohinger@astro.univie.ac.at

${ }^{1}$ On the 1 st of July 2002, the 100th extra-solar planet had been detected, moving around the sun-like star HD 2039. compared with Jupiter's distance from our Sun) around its hoststar 55 Cancri, which is the first extra-solar Jupiter-like planet moving in a similar orbit to Jupiter.

With the detection of more and more extra-solar planetary systems, general stability studies of orbits in such systems have become more and more important. On the one hand, there are many investigations - mainly with the aid of numerical experiments - for individual exo-solar systems, especially for those with multiple planetary companions like Ups Andromedae (see e.g. Lissauer 1999; Laughlin \& Adams 1999; Rivera \& Lissauer 2000; Stepinski et al. 2000; Laskar 2000; Barnes \& Quinn 2000; Jiang \& Ip 2000; Laughlin \& Chambers 2001; Kiseleva-Eggleton \& Bois 2001; Goździewski et al. 2001), Gliese 876 (see e.g. Laughlin \& Chambers 2001; Marcy et al. 2001; Kinoshita \& Nakai 2001; Rivera \& Lissauer 2001; Rivera \& Lissauer 2002; Goździewski et al. 2002; Hadjidemetriou 2002; Jianghui et al. 2002) or HD 82943 (see e.g. Goździewski $\&$ Maciejewski 2001; Hadjidemetriou 2002). These three planetary systems are of great interest, since the planets move in resonant orbits. 
On the other hand there are general stability studies of P- and S-type motions ${ }^{2}$ using the elliptic restricted three body problem ${ }^{3}$ like the ones by Harrington (1977), Szebehely (1980), Szebehely \& McKenzie (1981), Dvorak (1984, 1986) Rabl \& Dvorak (1988), and Dvorak et al. (1989). More recently these studies have been continued by Holman \& Wiegert (1999), Pilat-Lohinger (2000a,b) and Pilat-Lohinger \& Dvorak (2002). Since all these investigations are restricted to the planar motion of the planet we have no information on the influence of the inclination on the stable motion. This is the subject of this paper. Stability studies in double star systems are of great interest, not only since we know already 5 examples of S-type motion but also because of the fact that more than half of the stars form double or multiple systems.

In the following sections we present our stability study of P-type motion in a binary with mass-ratio ${ }^{4}(\mu)$ equal to 0.5 (i.e. the two stars have equal masses). For such a system there exist several studies of periodic orbits in the circular resticted three body problem. The first investigations for the three dimensional case date back to Goudas $(1961,1963)$, followed by the work of Markellos (1977, 1978), Michalodimitrakis (1979), Perdios \& Markellos (1988) and Zagouras (1977) and more recently Broucke (2001). In the present study we determine numerically (a) by means of orbital computations and (b) with the aid of the Fast Lyapunov Indicator ( $F L I)$ (Froesché et al. 1997) the variation of the stable zone by increasing the eccentricity of the binary from 0 to 0.5 . The computations are carried out for planar planetary orbits and inclined ones (for $i$ up to $50^{\circ}$ ).

In Sect. 2 we describe the initial conditions and their variations for the different numerical studies and we define the stability criterion. The results for the circular and for the elliptic problem will be discussed in Sects. 3 and 4, respectively. Section 5 is concerned with the escape times, which are given for all unstable orbits and finally in Sect. 6 we present our computations of the FLIs in the elliptic problem and a comparison with the results of the orbital computations.

\section{The numerical setup}

To establish the limiting curve for stable planetary orbits, we analyzed the results of the numerical integration of the three dimensional equations of motion of the elliptic restricted three-body problem using two integration methods (a) the Lieseries (see e.g. Lichtenegger 1984 and Hanslmeier \& Dvorak 1984) for the orbital computations and (b) the Bulirsch-Stoer method for the computation of the FLIs (this program was kindly provided by R. Gonczi from the Observatory of Nice). According to previous results - and contrary to the S-type orbits - the stability limits for P-types are almost independent of the mass-ratio of the primaries (see Dvorak et al. 1989). As a

\footnotetext{
${ }^{2}$ In double stars we distinguish 2 types of motion: planetary(P-)type motion, where the planet moves around both stars and satellite- (S-) type motion, where the planet moves around one of the two stars.

3 The elliptic restricted three body problem describes the motion of a massless body moving in the gravitational field of two massive bodies, which move in Keplerian orbits around their center of mass.

${ }_{4}$ The mass-ratio of two bodies $m_{1}$ and $m_{2}$ is given by $m_{2} /\left(m_{1}+m_{2}\right)$.
}

consequence we studied only the case of equal massive primary bodies.

To examine the phase space around such a binary with mass-ratio $(\mu)$ equal 0.5 , we varied the initial conditions in the following way:

1. for the orbits of the binary:

(a) the eccentricity $e$ was increased from 0 to 0.5 with $\Delta e=0.05$

(b) for the starting positions of the primaries we used the apo-astron and peri-astron (for $e>0$ );

2. for the planetary orbits:

(a) the inclination $i$ of the planetary orbit to the plane of motion of the binary was increased from $0^{\circ}$ to $50^{\circ}$ with a step size of $\Delta i=2.5^{\circ}$;

(b) the initial distance (A) of the planet from the barycenter of the binary was varied from 1.8 to either $3.5 \mathrm{AU}$ (for $e \leq 0.15$ ) or $4.5 \mathrm{AU}$ (for $e \geq 0.2$ ) with $\Delta \mathbf{A}=$ $0.05 \mathrm{AU}$ and

(c) for each $\mathbf{A}$ we used 4 starting positions for the planets which are defined by the following angles of the planetary orbit to the connecting line of the primaries: $\alpha=0^{\circ}, 45^{\circ}, 90^{\circ}$ and $135^{\circ}$.

All planetary orbits were initially circular with respect to the barycenter of the system. From the variations of the initial conditions it follows that we computed for all distances from the barycenter 8 (if $e>0$ ) or 4 (if $e=0$ ) planetary orbits. The stable motion is defined by the initial distances of the planets to the barycenter of the binary where all integrated orbits for a certain $\mathbf{A}$ are stable within the integration time. Therefore, the stable region depends on two parameters, namely on the eccentricity of the binary and on the inclination of the planet.

In total almost 50000 orbits were integrated for 50000 periods of the primaries. To study the dependence of the stable zone on $e$ and $i$ we analyzed all computed orbits and determined:

- an upper critical orbit (UCO) (see Dvorak et al. 1989), which forms the border of the stable zone and is therefore defined as the innermost orbit where all 8 (or 4 if $e=0$ ) different starting positions were found to be stable;

- a lower critical orbit (LCO) which forms the border of the unstable zone, since it is the outermost orbit where all 8 (or 4 if $e=0$ ) different starting positions were found to be chaotic;

- an escape time for the unstable orbits, which is given by the time when a close encounter with one of the primaries occurred (within the Hill's sphere).

From these studies it follows that we divided the phase space around the binary with $\mu=0.5$ into 3 regions: a stable zone, an unstable zone and in between a so-called mixed zone ${ }^{5}$, where both stable and unstable motion was found for a certain distance from the barycenter.

\footnotetext{
5 This zone was also called grey region or chaotic region in former studies (see Dvorak 1986).
} 


\subsection{The Fast Lyapunov Indicator}

In the second part of our study we used a fast method, the socalled Fast Lyapunov Indicator (Froeschlé et al. 1997), to distinguish between regular and chaotic motion. According to the definition of this indicator, which is simply the norm of the largest tangent vector $v_{i}$ :

$$
F L I(t)=\sup _{i}\left\|v_{i}(t)\right\| \quad i=1, \ldots, n
$$

( $n$ denotes the dimension of the phase space) it is obvious that chaotic orbits can be found very quickly because of the exponential growth of this vector in the chaotic region. Therefore, we needed only a few number of primary revolutions to determine the orbital behaviour.

In order to define the stable motion we introduced a critical value for the FLIs, which was set to $10^{7}$ according to the computed FLIs.

The computation of the FLIs has been done in principal for the same initial conditions as described above but with the following changes:

- we considered only the peri-astron positions of the binary;

- we took a finer grid in $\Delta \mathbf{A}$, namely $0.01 \mathrm{AU}$ and

- we reduced the computation time to 10000 periods of the binary.

\section{Results for the circular problem}

For this case we used a finer grid in the initial distance $\mathbf{A}$ $(\Delta \mathbf{A}=0.01 \mathrm{AU}-$ the same as for all FLI computations) for the orbital computations based on the Lie-series method which enables us to see a more detailed structure of the phase space at the borders between the different types of motion. Figure 1 shows a summary of the results of the circular problem in the $(\mathbf{A}, i)$ plane, where one can see the variation of the 3 types of motion: black for the stable, grey for the mixed and light grey for the unstable one with respect to the different initial inclinations of the orbital planes of the planets. In this case we see a nearly constant UCO (at $\mathbf{A}=2.25 \mathrm{AU}$ ) for inclinations up to $20^{\circ}$, and subsequently we observe an almost linear incresase of the stable zone, which starts at $\mathbf{A}=2.13$ AU for $i=50^{\circ}$. For the LCO we did not find a significant change with the inclination, which can be seen by the almost straight line at $\mathbf{A}=1.95 \mathrm{AU}$. The region between the $\mathrm{LCO}$ and $\mathrm{UCO}$ is the so-called mixed zone (where both motions can be found for a certain A), whose size is $0.3 \mathrm{AU}$ for all inclinations from $0^{\circ}$ to $15^{\circ}$. Thereafter, we recognize a separation of this zone due to a finger-like stable area from $i=15^{\circ}$ to $i=45^{\circ}$. A second finger-like separation due to another stable region occurs at inclinations between $25^{\circ}$ and $35^{\circ}$ which finally results in a large stable region close to the unstable zone from $i \geq 40^{\circ}$ on. Furthermore, for $i=47.5^{\circ}$ and $i=50^{\circ}$ a small completely unstable region appears at $\mathbf{A}=2.1 \mathrm{AU}$ so that the mixed region disappears almost completely for such large values of the initial inclinations. Recently, Broucke (2001) studied periodic orbits in a binary, where among others the family of direct orbits around both primaries with a radius 2.1 were investigated.

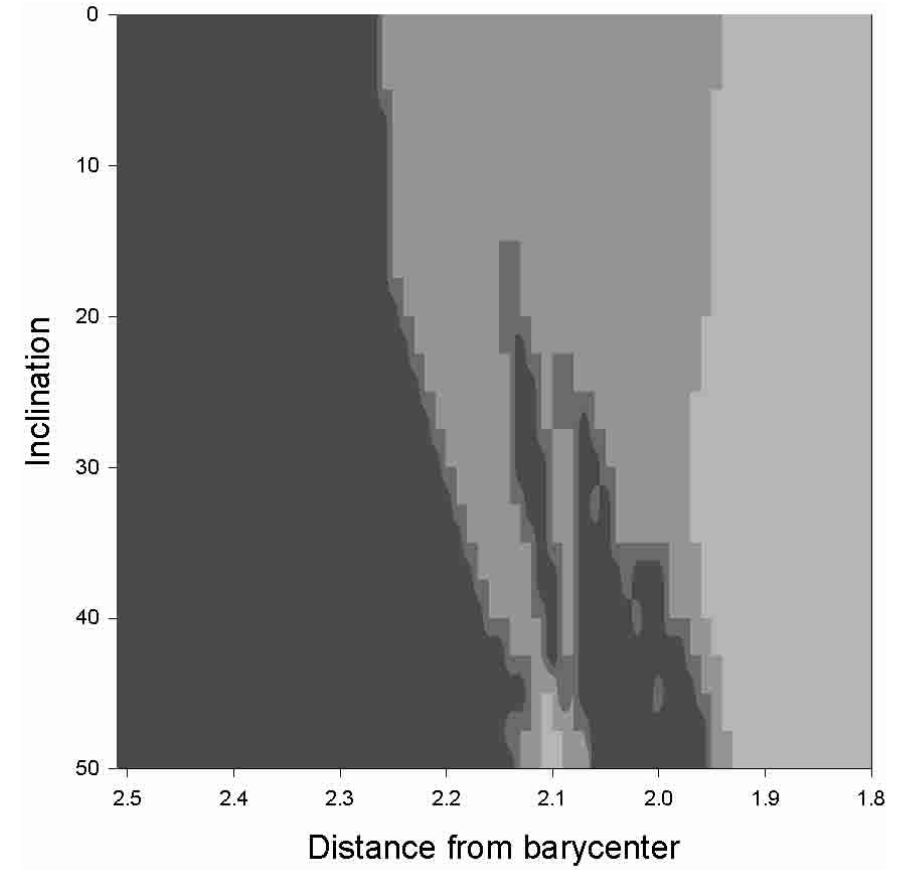

Fig. 1. The results of the circular problem presented in the $(\mathbf{A}, i)$ plane, where both axes are plotted from the highest to the lowest value, black shows the stable zone, white the unstable one and in between is a mixed region.

The stability of this L1v family (according to Hénon's terminolgy 1973) is not in contradiction to our results, since this distance belongs to the chaotic region (see Fig. 1), where the stable motion depends strongly on the initial position of the massless body. Moreover, for inclinations between $25^{\circ}$ and $45^{\circ}$ one can see a finger-like stable region at this distance.

In general the result shows an increase of the stable zone for higher inclinations. This seems to be in contrast to the study by Kozai (1962), who found more instability for high inclined orbits in the asteroid belt, which is caused by a resonance that pumps up the initial small eccentricities to large ones. But already for the outer Solar System it was found that the Kozai resonances plays only a role for orbits with high inclination and large eccentricity (see Thomas \& Morbidelli 1996). Since we study P-type orbits started in circular motion the Kozai resonance cannot be observed in our results.

In the "escape diagram" (Fig. 2) we plotted the inverse of the escape time ${ }^{6}$ for all orbits in the $(\mathbf{A}, i)$ plane. One can see clearly the border between the stable and the mixed zone. Also the fingerlike stable island within the mixed area at high inclinations is visible. In contrast thereto, it is difficult to distinguish between the mixed and unstable regions, due to the fact that the mean escape time at this border differs only by about 10 periods of the binary between the two types of motion.

The results of the circular problem obtained by using the Fast Lyapunov Indicator are summarized in Fig. 3, where we show again for each distance from the barycenter ( $x$-axis) and for the different inclinations ( $y$-axis) whether an orbit belongs

\footnotetext{
6 Since we have 4 starting positions for each set of $(\mathbf{A}, i)$, we took an average value for the escape times.
} 


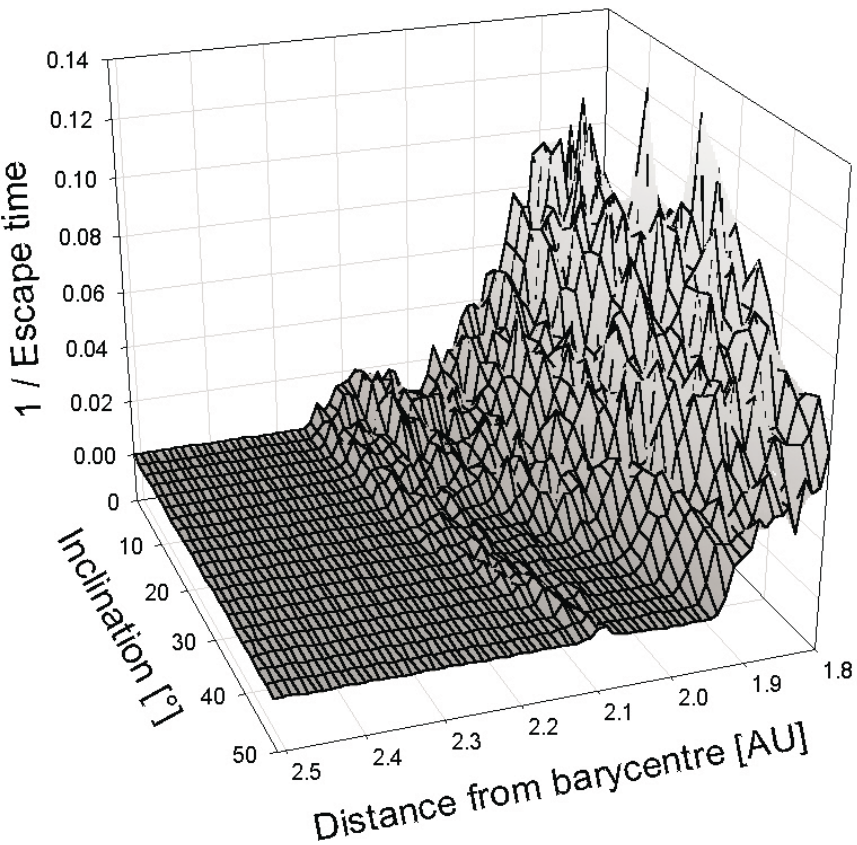

Fig. 2. The escape times computed for all orbits in the circular problem are summarized in the figure. One can see the distance of the planet from the barycenter on the $x$-axis, the different inclinations on the $y$ axis and the inverse of the escape time on the $z$-axis.

to the stable (black) to the mixed (grey) or to the unstable (white) zone. In general the result is in good agreement with that of the long-term orbital computations (Fig. 1). We see again almost constant zones for orbits with an inclination from $0^{\circ}$ to nearly $20^{\circ}$. The finger-like stable zone in the mixed area is similar to that of the orbital computations. The main difference is that the number of stable orbits in the mixed region found by means of the FLIs is smaller than that of the orbital computations for high inclinations. This is probably due to the fact that the computation of the FLIs indicates chaotic motion earlier. A new phenomenon found in this study is the appearance of a small strip of chaotic islands inside the stable region orientated parallel to the contour of the UCO. This may be caused by a mean motion resonance between the primaries and the planet, which will be studied more in detail in a further investigation.

\section{The variation of the $L C O$ and UCO in the elliptic problem}

In the elliptic problem we studied 10 different models: $0.05 \leq$ $e \leq 0.5$ (with $\Delta e=0.05$ ), for which we used only a grid of $0.05 \mathrm{AU}$ for the distance from the barycenter. Because of the ellipticity of the binary we have now to integrate 8 initial positions for a given $\mathbf{A}$, since the computations have been done starting with the binary in the peri-astron and in the apo-astron.

The results for the 10 elliptic models are given in Figs. 4-6; where we show for each eccentricity of the binary (from $e=0$ to 0.5 with $\Delta e=0.05$ ) two figures: a 3D plot for the escape times and a contour plot which displays the 3 zones of the different types of motion. Looking at the border between unstable and mixed motion, which is given by the computations of

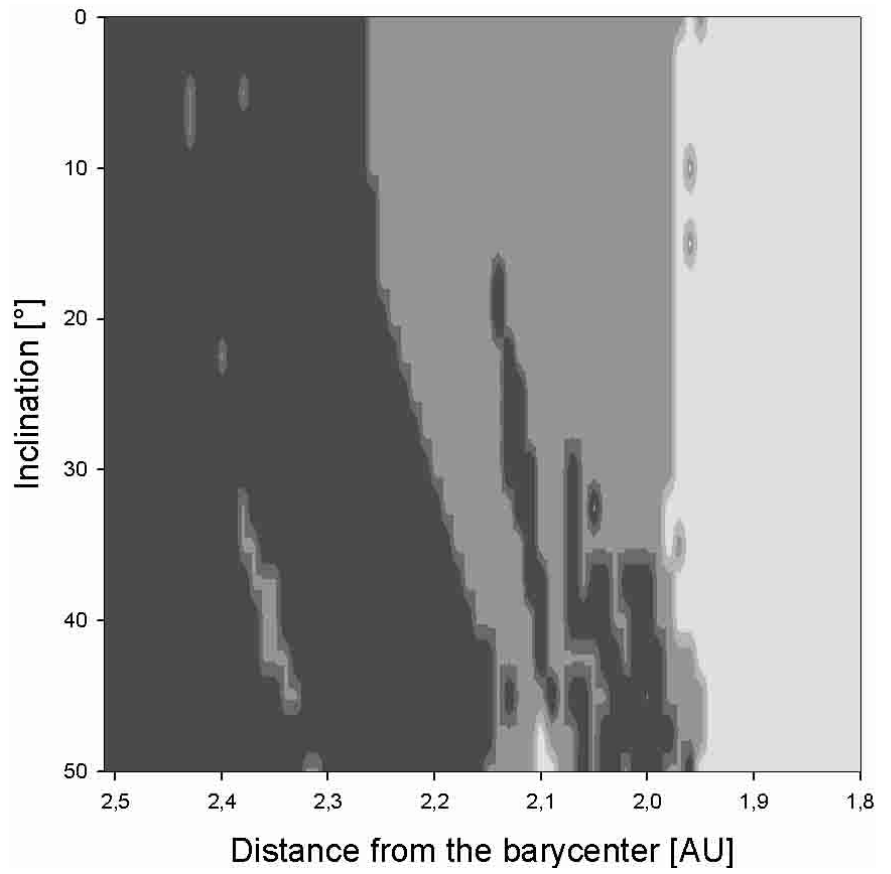

Fig. 3. The results of the FLI computation for the different distances to the barycenter ( $x$-axis) and the different inclinations ( $y$-axis). Note that the scaling of the axes in both directions is from the largest to the lowest value. The different shades represent the different types of motion: from black for small values of the FLIs (which corresponds to stable motion) to white for very large values of the FLIs (which corresponds to unstable region of Fig. 1).

the LCO, one can recognize that it remains nearly constant for a given eccentricity, with two exceptions: for $e=0.1$ at high inclinations, where a small island of mixed motion can be found around $\mathbf{A}=2.3 \mathrm{AU}$ and for $e=0.3$ which shows a variation of this border with the inclination. Furthermore, one can see a shift outwards (to larger A values) when we increase $e$. This behaviour is visible when increasing $e$ from 0 to 0.1 (Fig. 4: first three contour plots from the top) and again from $e=0.25$ to 0.35 (Fig. 5: last three contour plots), in the other cases this shift is much smaller.

The border of the stable motion which is given by the UCO shows an interesting appearance of unstable "fingers" starting at high inclinations (Fig. 4: second contour plot from the top), which become larger in the direction of zero inclination (Fig. 4: last two contour plots) when increasing the binary eccentricity. Then they split up the stable region and finally the border of the stable zone is shifted outward to a larger value of $\mathbf{A}$ (Fig. 5: contour plot on the top). This development is visible from $e=0.05$ to $e=0.2$ and again from $e=0.2$ to $e=0.5$, where in the second appearance the stable island remains longer within the mixed zone.

In the three dimensional graph of the LCO (Fig. 7), which is given by the initial distance from the barycenter of the last unstable orbit in the $(e, i)$-plane, one can observe an increase of the LCO for an eccentricity up to 0.1 with a more or less constant behaviour with respect to the inclination (from $\mathbf{A}=1.9$ to 2.5 AU). Between $e=0.1$ and 0.3 we find a slightly inclined plateau to larger A values (from 2.5 to $2.75 \mathrm{AU}$ ). A further 

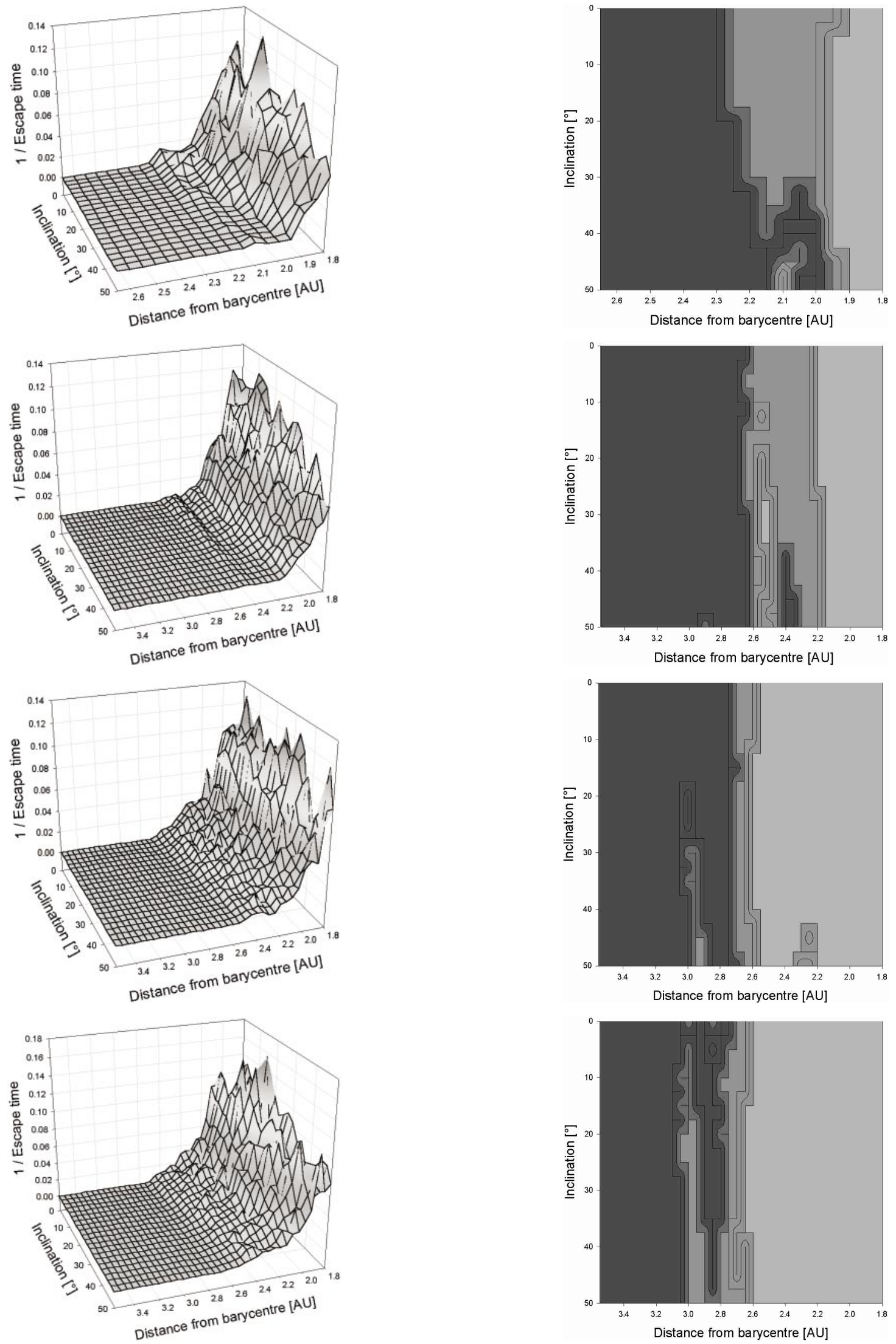

Fig. 4. The panels on the left side show the inverse of the escape time in the (A, $i)$-plane and those on the right side show the stable zone (black), the mixed zone (grey) and the unstable zone (white) for $0 \leq e \leq 0.15$ (all for the same grid of initial conditions). 

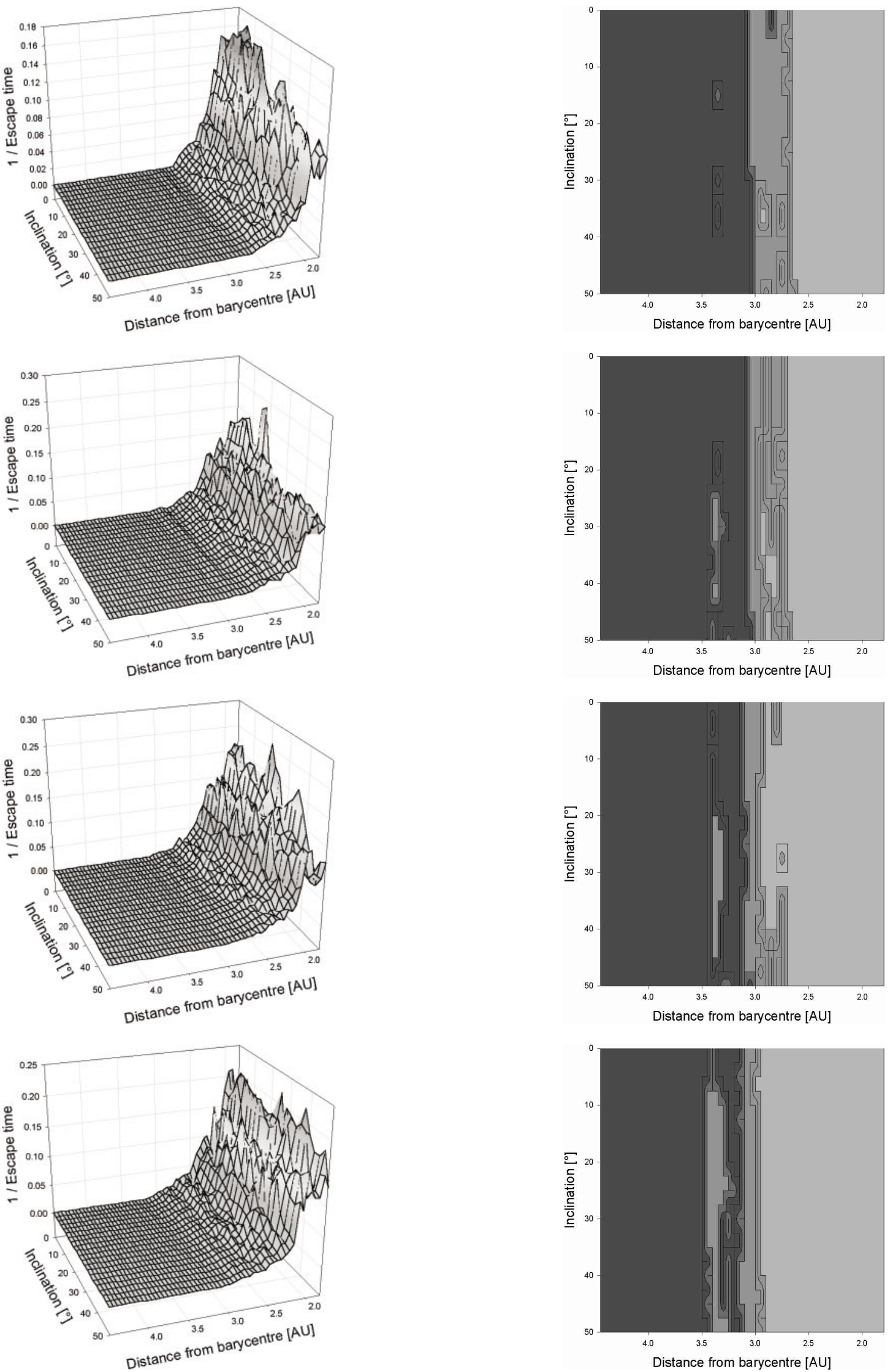

Fig. 5. The same figure as Fig. 4, but for $0.2 \leq e \leq 0.35$. 

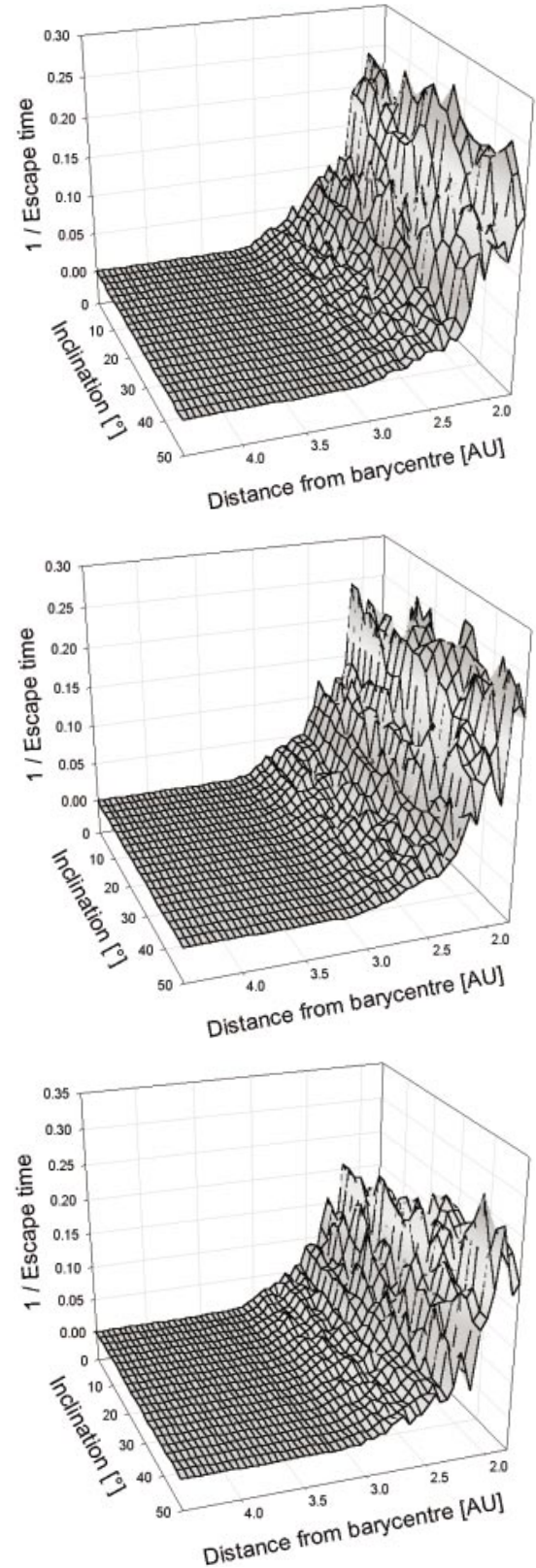

Fig. 6. The same figure as Fig. 4, but for $0.4 \leq e \leq 0.5$.

increase of $e$ up to 0.35 yields a jump in $\mathbf{A}$ and subsequently $\mathbf{A}$ neither depends on $e$ nor on $i(\mathbf{A}=2.95 \mathrm{AU})$.

The behaviour of the UCO (Fig. 8), which shows the dependence of the border of the stable zone on the eccentricity of the binary and on the inclination of the planet's orbit, is somewhat similar to the LCO. We observe for small eccentricities $(e \leq 0.1)$ a weak dependence on the inclination of the planetary orbit. Between $e=0.1$ and 0.3 one can see some irregularities and three small plateaus - one for high inclinations between $40^{\circ}$ and $50^{\circ}$, the second around $i=30^{\circ}$ (both for $e$ from 0.1 to 0.2 ) and the third for low inclined orbits $\left(i<15^{\circ}\right)$ and $e$ between 0.15 and 0.25 . The irregularities between $e=0.15$ and 0.3 cause jumps of the UCO followed by a big plateau, where neither $e$ nor $i$ will change the UCO in this region (for $e \geq 0.3$ ). Furthermore, the figure shows a "hill
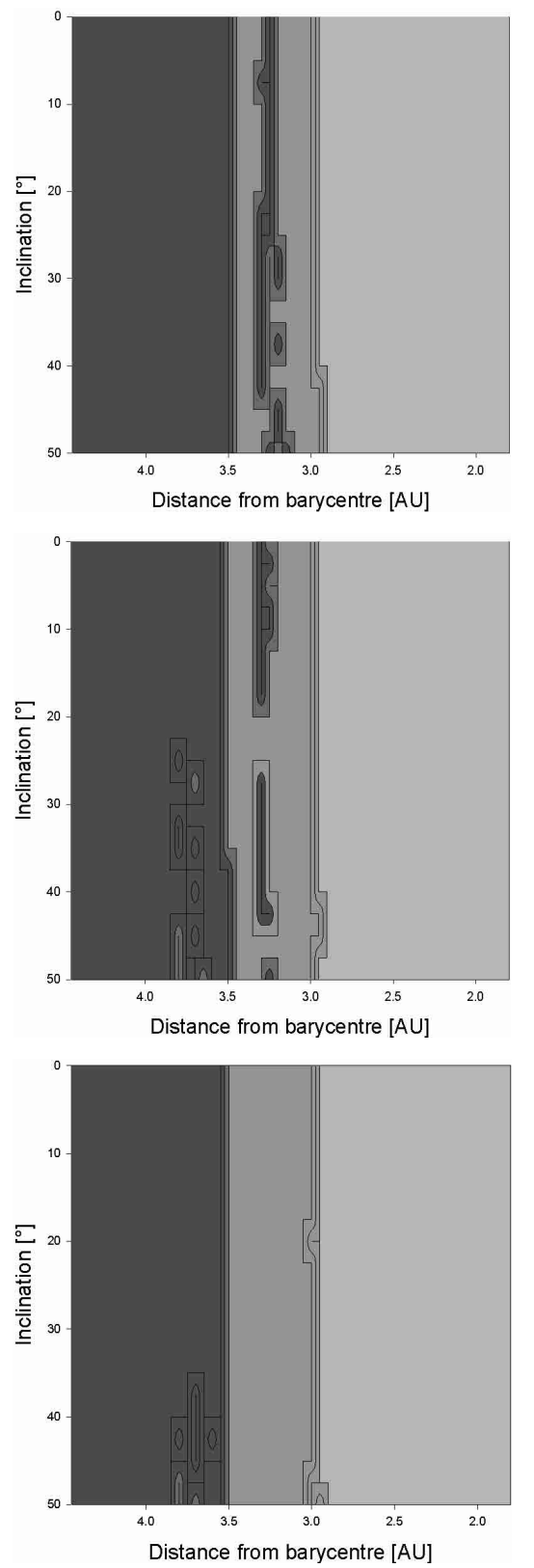

structure" for high eccentricities $(e \geq 0.4)$ and high inclinations $\left(i \geq 25^{\circ}\right)$.

\section{The escape times}

From the orbital computations we determined the escape times, which are defined by the moment of a close encounter of the planet with a primary. The three dimensional plots (Figs. 4-6: left) show the inverse of the mean value of the escape times (calculated from all 8 (or 4 if $e=0$ ) orbits for a certain distance from the barycenter). Globally, we can say that the border between stable and mixed motion is much more visible than that between mixed and unstable motion - this is again due to the fact that the escape times near the LCO of the two motions do not differ very much. From the limit of the stable zone, one can see a steep increase in the $3 \mathrm{D}$ plots, which means that a 


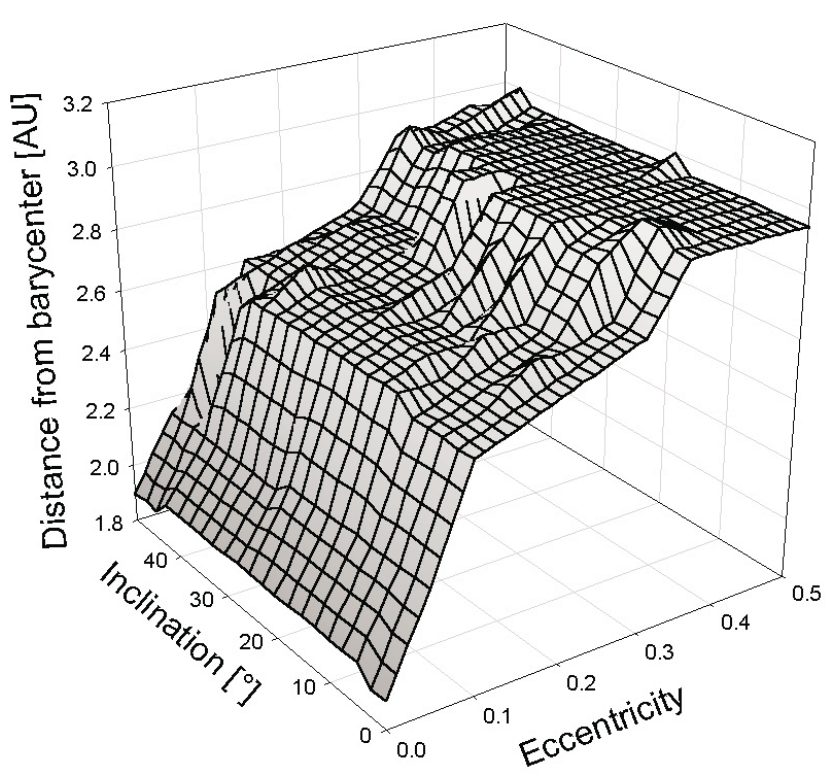

Fig. 7. Global result of the $\mathrm{LCO}$ in the $(e, i)$-plane.

closer initial distance to the binary leads to an earlier escape of the planet. An analysis of the innermost computed orbit (i.e. $\mathbf{A}=1.8 \mathrm{AU}$ ) shows that the higher the eccentricity and the inclination the faster an escape occurs.

\section{Results of the elliptic problem by means of the FLIs}

In this study we considered only the orbits where the primaries were started in the peri-astron, so that the comparison of the results shows not only differences due to the different integration methods but also due to the fact that we ignored the apoastron starting positions. The computations have been carried out for 10000 periods of the primaries. In order to distinguish between the different types of motion we took the same critical value for the FLIs as in the circular problem (i.e. $10^{7}$ ) to define the border of the stable motion.

The calculation of 4 orbits for each distance from the barycenter - due to the variation of $\alpha$ (as described in Sect. 2) leads again to 3 types of motion: the stable, the mixed and the unstable ones, which are represented by different grey colors in the figures. The study of the FLIs has been carried out to confirm the results of the orbital computations, especially the size of the stable region. Therefore, we show two examples for the elliptic case (Fig. 9 for $e=0.1$, and Fig. 10 for $e=0.5$ ), since the differences of the two studies for the other eccentricities are similar to those shown in Figs. 9 and 10. In general, we can say that there is no strong influence of the inclination on the zones of the different types of motion. The main differences between the two studies occur due to the fact that we used a finer grid in $\mathbf{A}$ for the computations of the FLIs, which gives a more detailed structure of the phase space.

The results of the FLIs show also the shift of the mixed and the stable zones outwards, i.e. to larger distances form the

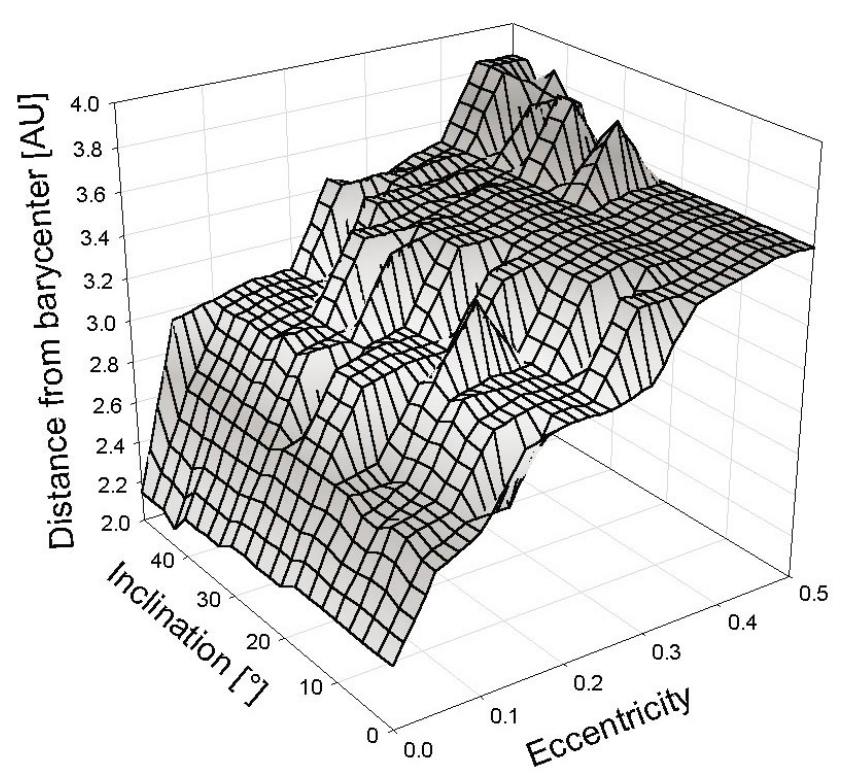

Fig. 8. Global result of the UCO in the $(e, i)$-plane.

barycenter when we increase the eccentricity. More precisely, the border between unstable and mixed motion (i.e. the LCO) is shifted from $\mathbf{A}=1.95 \mathrm{AU}$ (for $e=0$ ) to $2.18 \mathrm{AU}$ (for $e=0.05$ ) and to distances between 2.6 and 2.67 AU depending on the inclination for $e=0.1$ (Fig. 9). Thereafter the limit remains at this position up to $e=0.3$. For an eccentricity of $e=0.35$ we observe another shift to about $\mathbf{A}=3 \mathrm{AU}$ which corresponds to the position of a chaotic strip in the stable region for $e=0.1$ (Fig. 9). This chaotic strip is the main difference between the two studies, since it ends at about $i=30^{\circ}$ in the plot of the orbital computations (Fig. 5 - third contour plot). Therefore, we have the same border between stable and mixed motion for the high inclined orbits, while for orbits with $i<30^{\circ}$ the UCO varies of about $0.3 \mathrm{AU}$ for the two studies. A further increase of the eccentricity causes that the stable zone between the LCO and UCO gets smaller and smaller, which finally leads to a shift of the two borders. Additionally, another chaotic island appears at about 3.4 AU from the barycenter - a first indication can already be seen in Fig. 9 for $i=30^{\circ}$, while in the results of the orbital computations it appears for the first time for $e=0.2$.

The second example for the elliptic case $(e=0.5)$ shows the same border between unstable and mixed motion, while the stable zone begins beyond 3.8 AU from the barycenter which is about $0.3 \mathrm{AU}$ farther outside than in the result of the orbital computations - except for high inclinations $\left(i \geq 47.5^{\circ}\right)$, where the UCO is the same in both studies. The finer grid in A shows again a more detailed structure of the phase space (which seemed to be without structure in the results of the orbital computations - Fig. 6 last contour plot).

\section{Conclusions}

Up to now there are no known planets in binaries moving around both stars but from the cosmogonic point of view this scenario cannot be excluded. Certainly, for a stability study in 


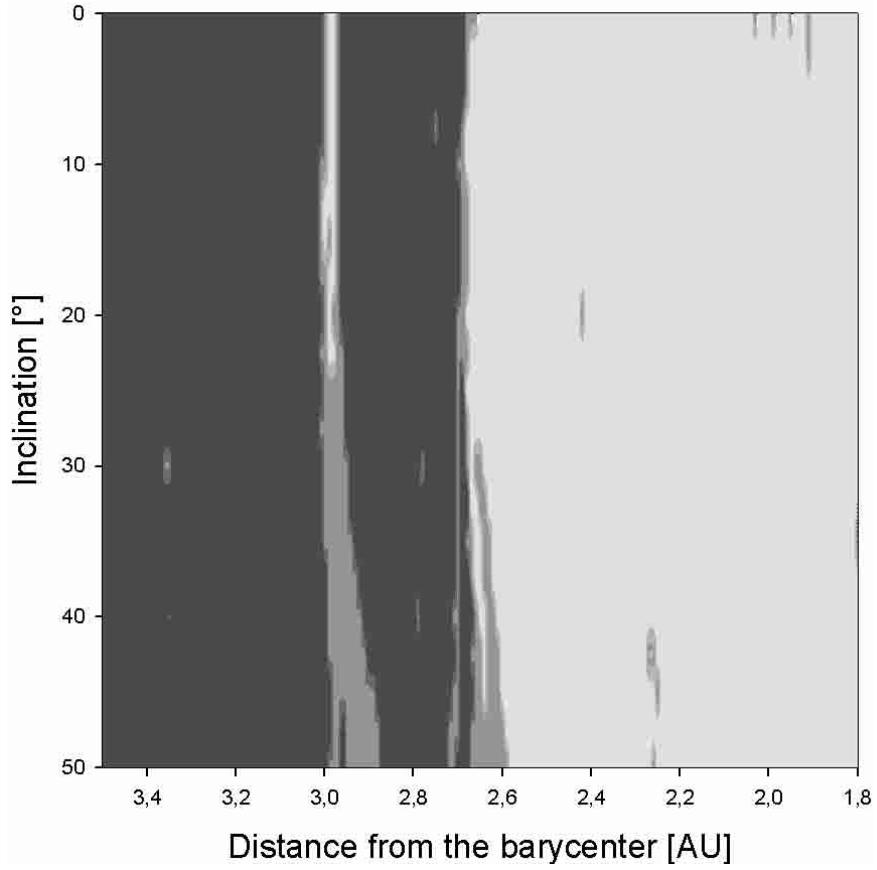

Fig. 9. Global result of the $F L I$ computation when the two components of the double star move in low eccentric orbits $(e=0.1)$. The different distances to the barycenter are plotted on the $x$-axis and the different inclinations are shown of the $y$-axis. Note that the scaling of the axes in both directions is from the largest to the lowest value. The different shades represent the different types of motion: from black for stable motion to white for unstable motion.

the habitable zone (i.e. at distances from the star where liquid water may exist) it is more interessting to study S-type orbits.

To tackle the problem of stability of P-type motion in a binary, we did numerical experiments in the framework of the spatial elliptic restricted three body problem. The new results concern the stability of inclined orbits with respect to the orbit of the binary. Two different series of numerical integrations of the equations of motion were analyzed. For a simple analysis of the stability (stable within the integration time without a close encounter with one of the primaries) we integrated with the Lie-series method almost 50000 orbits for 50000 periods of the binary and checked their stability or instability, so that the phase space was divided into 3 regions (stable, mixed and unstable one) by the determination of an Upper Critical Orbit (UCO) and a Lower Critical Orbit (LCO). Furthermore, the escape time was computed for each orbit. The respective diagrams were compared and were found to be in good agreement. Additionally, in another numerical integration using the Bulirsch-Stoer method we computed the Fast Lyapunov Indicator $(F L I)$ which was used as chaos indicator.

Summarizing, we can say the following:

- the stability limit varies between 2.1 AU and 3.85 AU depending on the eccentricity of the binary;

- for some eccentricities only we found a clear dependence of the stability limits on the inclination of the planetary orbit

- the regions of stable, mixed and unstable orbits show the following behaviour: for certain eccentricities a small finger-like unstable island appears at high inclinations and

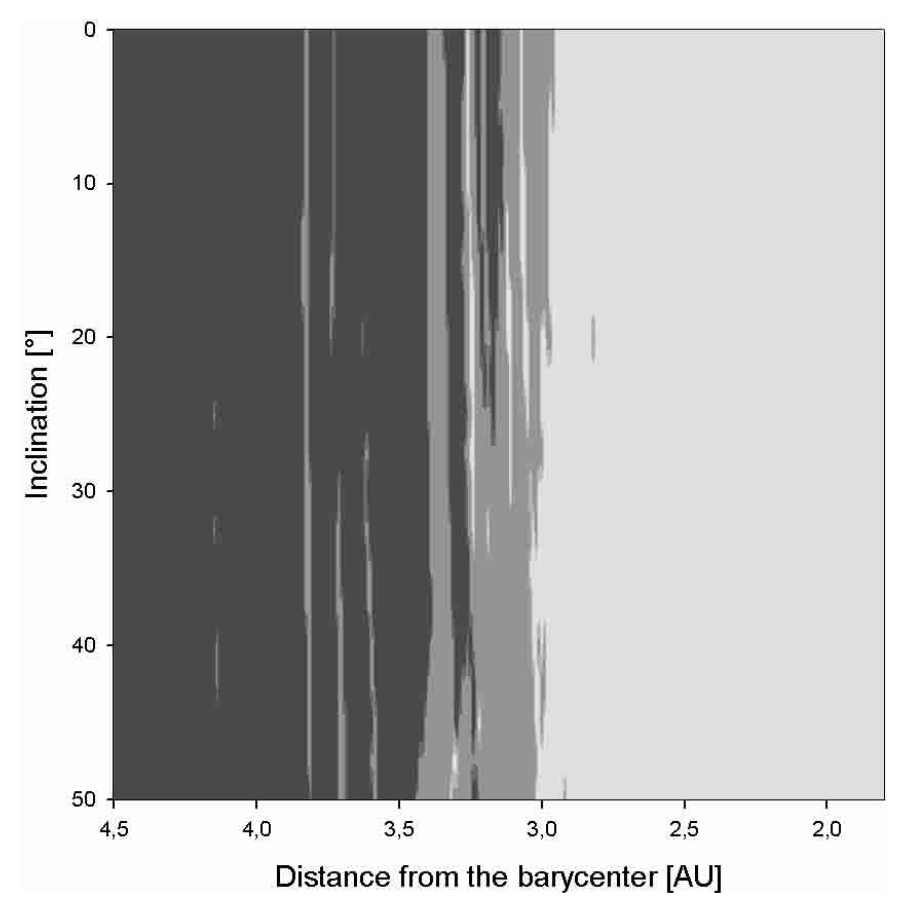

Fig. 10. Same as Fig. 9 for $e=0.5$.

evolves to a small strip of instability in the $(\mathbf{A}, i)$ plots if we increase the binary's eccentricity. This developement leads finally to a shift of the stable zone to larger distances from the barycenter (this can be seen from $e=0.05$ to $e=0.2$ and repeats for eccentricities from 0.2 to 0.5 );

- the computations of the FLIs show in principal the same result, only the unstable islands in the stable zone appear earlier and evolve faster to unstable strips so that the border of the stable zone is shifted outwards earlier. Of course the plots show a more detailed structure due to the finer grid in the distance from the barycenter. Differences occur also due to the reduced integration time - sometimes there are small islands of mixed motion in the unstable zone (Fig. 9) or stable motion was found in the mixed zone (Fig. 10);

- an interessting phenomenon was found in the result of the circular problem obtained by the computations of the FLIs, which shows a strip of chaotic behaviour in the stable region (for high inclinations). This is probably due to a resonance, which has to be studied in more detail, since it could not confirmed with long-term integrations using the Lie-series method for $10^{6}$ periods of the binary.

As a next step, we will investigate the detailed structure of the resonances of the P-type motion in binaries, and we will study how the three zones will be influenced by a massive planet (in the different models), which leads to secular resonances.

Acknowledgements. We thank the referee for suggestions to improve our paper. This work was carried out within the framework of the FWF project No. P14375-TPH. One of the authors (EP-L) was financed by the Hertha-Firnberg-Project T122. 


\section{References}

Barnes, R., \& Quinn, T. 2001, ApJ, 550, 884

Broucke, R. A. 2001, CMDA, 81, 321

Dvorak, R. 1984, CMDA, 34, 369

Dvorak, R. 1986, A\&A, 167, 379

Dvorak, R., Froeschlé, Ch., \& Froeschlé, C. 1989, A\&A, 226, 335

Froeschlé, C., Lega, E., \& Gonczi, R. 1997, CMDA, 67, 41

Gozdziewski, K., Bois, E., Maciejewski, A. J., \& Kiseleva-Eggleton, L. 2001, A\&A, 378, 569

Gozdziewski, K., \& Maciejewski, A. J. 2001, A\&A, 563, L81

Gozdziewski, K., Bois, E., \& Maciejewski, A. J. 2002, MNRAS, 332, 839

Goudas, C. L. 1963, Icarus, 2, 1

Hadjidemetriou, J. 2002, CMDA, 83, 141

Hanslmeier, A., \& Dvorak, R. 1984, A\&A, 132, 203

Harrington, R. S. 1977, AJ, 82, 753

Hénon, M., A\&A, 28, 415

Holman, M. J., \& Wiegert, P. A. 1999, AJ, 117, 621

Jiang, I., \& Ip, W. 2001, A\&A, 367, 943

Jianghui, J., Guangyu, L., \& Lin, L. 2002, ApJ, 572, 1041

Kinoshita, H., \& Nakai, H. 2001, Publications of the Astronomical Society of Japan, 53, L25

Kiseleva-Eggleton, L., \& Bois, E. 2001, ApJ, 553, L73

Kozai, Y. 1962, AJ, 67, 591

Laskar, J. 2000, Phys. Rev. Lett., 84, 3240
Laughlin, G., \& Adams, F. C. 1999, ApJ, 526, 881

Laughlin, G., \& Chambers, J. E. 2001, ApJ, 551, L109

Lichtenegger, H. 1984, CMDA, 34, 357

Lissauer, J. J. 1999, Nature, 398, 659

Marcy, G., Butler, R. P., Fischer, D., et al. 2001, ApJ, 556, 296

Marcy, G., Butler, R. P., Fischer, D. A., et al. 2002, ApJ, 581, 1375

Markellos, V. V. 1977, MNRAS, 180, 103

Markellos, V. V. 1978, MNRAS, 184, 273

Michalodimitrakis, M. 1979, A\&A, 76, 6

Pilat-Lohinger, E. 2000a, Proc. of the 5th Alexander von Humboldt Colloq. on Celestial Mechanics, ed. R. Dvorak, \& J. Henrard (Kluwer Academic Publisher), 329

Pilat-Lohinger, E. 2000b, Proc. of the 2nd Austrian Hungarian Workshop on Trojans and related Topics, ed. F. Freistetter, R. Dvorak, \& B. Erdi (Eötvös University Press), 77

Pilat-Lohinger, E., \& Dvorak, R. 2002, CMDA, 82, 143

Perdios, E., \& Markellos, V. V. 1988, CMDA, 42, 187

Rabl, G., \& Dvorak, R. 1988, A\&A, 191, 385

Rivera, E. J., \& Lissauer, J. J. 2000, ApJ, 530, 454

Rivera, E. J., \& Lissauer, J. J. 2001, ApJ, 558, 392

Stepinski, T., Malhotra, R., \& Black, D. C. 2000, ApJ, 545, 1044

Szebehely, V. 1980, CMDA, 22, 7

Szebehely, V., \& McKenzie, R. 1981, CMDA, 23, 3

Thomas, F., \& Morbidelli, A. 1996, CMDA, 64, 209

Zagouras, C. G., \& Markellos, V. V. 1977, A\&A, 59, 79 